\title{
Research progress on the central mechanism underlying regulation of visceral biological rhythm by per2 (Review)
}

\author{
LITING ZHU ${ }^{1}$, JUN YU ${ }^{2}$, WENYI ZHANG ${ }^{3}$, BIN XIE $^{4}$ and YI ZHU ${ }^{5,6}$ \\ ${ }^{1}$ Department of Integrative Medicine, School of Basic Medical Sciences, Fudan University, \\ Shanghai 200032; ${ }^{2}$ Department of Rehabilitation, Wuxi Hand Surgery Hospital, Wuxi, Jiangsu 214000; \\ ${ }^{3}$ Zhongshan Rehabilitation Branch, Jiangsu Provincial People's Hospital; ${ }^{4}$ The 1st Clinical College, \\ Nanjing University of Chinese Medicine; ${ }^{5}$ Department of Rehabilitation, Second School of Clinical Medicine, \\ Nanjing University of Chinese Medicine, Nanjing, Jiangsu 210046; ${ }^{6}$ Department of Acupuncture and Rehabilitation, \\ Jiangsu Provincial Second Hospital of Traditional Chinese Medicine, Nanjing, Jiangsu 210017, P.R. China
}

Received November 30, 2013; Accepted April 25, 2014

DOI: $10.3892 / \mathrm{mmr} .2014 .2559$

\begin{abstract}
The period circadian clock 2 (per2) gene plays an important role in modulating the circadian rhythm in the central nervous system. Its protein product, PER2, is mainly expressed in the suprachiasmatic nucleus (SCN) and limbic system, including the central nucleus of the amygdala (CeA), the bed nucleus of the stria terminalis (BNST) and the hippocampus. PER2 rhythmic expression regulates hypothalamus-pituitary-adrenal (HPA) axis excitability and circadian rhythm via integration of optical signals and corticotropin-releasing factor (CRF) stress-related neurotransmitters, resulting in circadian rhythmicity in target organs. Moreover, glucocorticoids and glucocorticoid receptors exert strong negative feedback to the HPA axis and certain regions of the limbic system, modulating rhythmic per 2 expression in peripheral organs. To date, the mechanism of action of PER2 in the limbic system and the HPA axis remains unclear, yet the per 2 gene is considered valuable in clinical research for the study of metabolic syndromes, functional gastrointestinal disorders and certain liver diseases. In this review, we summarize the biological effects of the per 2 gene and its protein product, PER2, in the limbic system, its involvement in regulation of the HPA axis by the limbic system and the resulting effects on the biological rhythm of target organs, and its clinical significance.
\end{abstract}

Correspondence to: Dr Yi Zhu, Department of Acupuncture and Rehabilitation, Jiangsu Provincial Second Hospital of Traditional Chinese Medicine, no. 138 Xianlin Avenue, Nanjing, Jiangsu 210017, P.R. China

E-mail: zhuyi1010@yeah.net

Key words: CLOCK gene, limbic system, corticotrophin-releasing factor, glucocorticoid, circadian rhythm

\section{Contents}

1. Introduction

2. Biological features of per 2

3. Effects of PER2 and corticotropin-releasing factor (CRF) on visceral circadian rhythm motion in the limbic system

4. The PER2-hypothalamus-pituitary-adrenal (HPA) axis interaction regulates visceral activities

5. Clinical significance of the PER2-HPA axis interaction on regulation of visceral rhythmic movement

6. Perspectives

\section{Introduction}

The Circadian Locomotor Output Cycles Kaput (CLOCK) and other genes, including brain and muscle ARNT-like protein 1 (bmall), period circadian clock (per) $1 / 2 / 3$, cryptochrome (cry) $1 / 2$, nuclear receptor subfamily 1 , group D, member 1 (NRIDI, also known as rev-erb $\alpha$ ), and differentiated embryonic chondrocyte gene (dec) $1 / 2$, predominantly participate in circadian rhythm feedback and regulation of clock-controlled genes in a feed-forward loop (1), accounting for $99 \%$ of the regulation. In humans, the CLOCK gene is crucial for maintaining a 24-hour circadian rhythm in behavior, physiology and the endocrine system $(2,3)$. The CLOCK gene is mainly expressed in specific groups of the suprachiasmatic nucleus (SCN), the ventromedial hypothalamus, olfactory bulb, amygdala, lateral habenula, hippocampus and hypothalamus $(4,5)$. The per 2 gene is expressed in the central and peripheral nervous systems and operates as a pacemaker of circadian rhythm in the brain of humans and other mammals, regulating spontaneous activity, metabolism and behavior. Rhythmic per 2 expression is regulated by the $\mathrm{SCN}$, but also, via negative feedback regulation from the bed nucleus of the stria terminalis (BNST) and the central nucleus of the amygdala (CeA) in the limbic system, as well as from corticosterone (6-8). Per2 is crucial for regulating visceral cyclic rhythmic activities through the HPA axis of the limbic system (6-8). Current research has addressed the mechanism 
of action of PER2 on target organs, the HPA axis and the limbic system (6-8). However, it remains poorly understood whether per 2 is involved in regulatory mechanisms of the limbic system on lower-level brain structures. Moreover, the relationship between functional areas of the limbic system is still unclear. In this review, we summarize per 2 biological features, what is known on its participation in limbic system regulation of the HPA axis, and on its effects on target organs.

\section{Biological features of per2}

The human per2 gene maps to chromosome 2, at 2q37.3 (gene ID: 8864 ), and is 51,529 base pairs (bp) long. The per 2 mRNA is 6,342 bp and contains 23 exons (http://www.ncbi.nlm.nih. gov/gene/8864); its transcription and translation give the PER2 protein, which contains two PER-ARNT-SIM (PAS) domains (PAS-A and PAS-B) to mediate homo-mPER interactions and interactions with transcription factors (9). The per2 gene is a member of the Period gene family. PER2-immunoreactive (ir) nuclei reaches the peak in expression at ZT13 (9 p.m.) and down to the lowest level of expression at ZT1 (9 a.m.) (10).

PER2 is strongly associated with the biological functions of the CLOCK/BMAL1 complex. BMAL1 and CLOCK are members of the basic helix-loop-helix/PAS family of transcription factors (11). The regulation of circadian periodicity involves an induction of transcription and translation of perl, per 2 and per3 genes by the CLOCK/BMAL1 complex; these genes inhibit CLOCK/BMAL1-induced transcription, serving as a determinant of negative regulation (Fig. 1). However, only per 2 can regulate rhythmic periodicity and its amplitude in the central and peripheral nervous systems $(12,13)$, although its regulatory mechanism in the central nervous system is complex (14,15). Per2-ir neurons receive neuronal projections from the SCN and the sympathetic nervous system and are regulated by the neuroendocrine system via corticosterone, melatonin and adrenaline (14,15). Per2 is mainly expressed in the oval nucleus of the BNST and central nucleus of the amygdala $(\mathrm{CeA})$ in the limbic system. These nuclear groups regulate motion and emotion and exhibit rhythmic alterations $(16,17)$. Studies of the molecular mechanisms underlying PER2 functions have shown that cyclic adenosine monophosphate, protein kinase $\mathrm{A}$ and $\mathrm{C}$, and mitogen-activated protein kinase are involved in the intracellular signaling pathway regulated by PER2 in the central and peripheral nervous systems. Moreover, per 2 cyclical variation is directly regulated by the SCN and its lower-level brain structures (Fig. 2) (18-20).

\section{Effects of PER2 and corticotropin-releasing factor (CRF) on visceral circadian rhythm motion in the limbic system}

Biological function of PER2 in the limbic system. Rhythmic expression of the PER2 protein results from rhythmic transcription-translation of the per2-CLOCK genes. The PER2 protein is ideal for observing rhythmic changes of the per2 gene (21). The BNST, CeA, basolateral amygdala and hippocampal dentate gyrus (DG) are four regions expressing the PER2 protein in the rat limbic system $(4,5)$. These regions are important neuroendocrine areas, involved in motion, emotion, behavior and mental adjustment during stress, which indicates that per 2 in the limbic system may be involved in stress- and drug abuse-related behavior and neuroendocrine responses, regulation of ingestion, learning and memory, maternal behavior and reproduction, and may overally target multiple organs (22-24). Rhythmic changes in PER2 expression show reverse profiles in different brain regions. Within the BNST and CeA, PER2 expression is maximal during the transition between day and night and almost synchronized with PER2 expression in the SCN. However, in the basolateral amygdala and hippocampal DG, PER2 expression changes are opposite to those observed in the SCN (Fig. 3). Previous studies have shown region-specific periods of PER2 in different brain sites of the limbic system (25), which may be associated with the negative feedback of PER2 to the CLOCK-BMAL1 dimer, which suppresess its own expression (26).

Similar patterns of PER2 rhythmic expression in the limbic system and SCN. PER2 alterations in the SCN are strongly associated with PER2 expression in the limbic system. Bilateral SCN lesions, or long-term 24-hour room illumination, can eliminate PER2 rhythmic expression in the SCN, resulting in a behavioral rhythm disorder $(16,17)$. Rhythmicity of PER2 expression is also completely eliminated in the BNST, CeA, basolateral amygdala, DG and their lower-level brain structures $(16,17)$. Unilateral SCN lesions do not affect circadian rhythm-related behavior, but attenuate the significance of PER2 rhythmic expression in the ipsilateral limbic system (16), suggesting similar patterns of PER2 rhythmic expression and the presence of an important neuronal link between the SCN and specific limbic system areas (27).

$C R F$ regulates PER2 in the limbic system and the hypothalamus. CRF is abundantly expressed in the hypothalamic paraventricular nucleus. CRF is a promoter of and is activated by, the HPA axis during stress. In the paraventricular nucleus, $\mathrm{CRF}$ is regulated by GABAergic neurons that are mediated by $\mathrm{CRF}$ and other neurotransmitter projections from higher level brain structures, including the prefrontal cortex, hippocampus, nucleus accumbens septi, CeA and BNST (Fig. 5) (28). There is a strong interaction between dopamine and CRF, and this interaction regulates PER2 expression and its rhythmic changes (29). The CeA and BNST receive dopamine signals (30) and are enriched in CRF-ir neurons (31-33). Unilateral 6-hydroxydopamine injury of dopaminergic neurons in the medial forebrain bundle causes reduced PER2 expression in the ipsilateral BNST and CeA, and also decreased expression of CRF neurons $(34,35)$. Following unilateral $C R F$ gene silencing in the BNST and CeA, ipsilateral PER2 expression almost disappears. However, there is no co-expression of CRF and PER2 (10), indicating that the regulatory effect of intracerebral CRF on PER2 possibly depends on intracellular signal transduction pathways.

Glucocorticoids regulate rhythmic PER2 expression in the BNST and CeA. PER2 expression in the BNST and CeA is synchronized with rhythmic PER2 alterations in the SCN affected by glucocorticoids. Circadian corticosterone release is regulated by the $\mathrm{SCN}$, leading to rhythmic per2 mRNA expression in hepatocytes and fibroblasts $(36,37)$. Glucocorticoid receptors are expressed in the BNST, CeA, basolateral amygdala and hippocampal DG. Endogenous and 


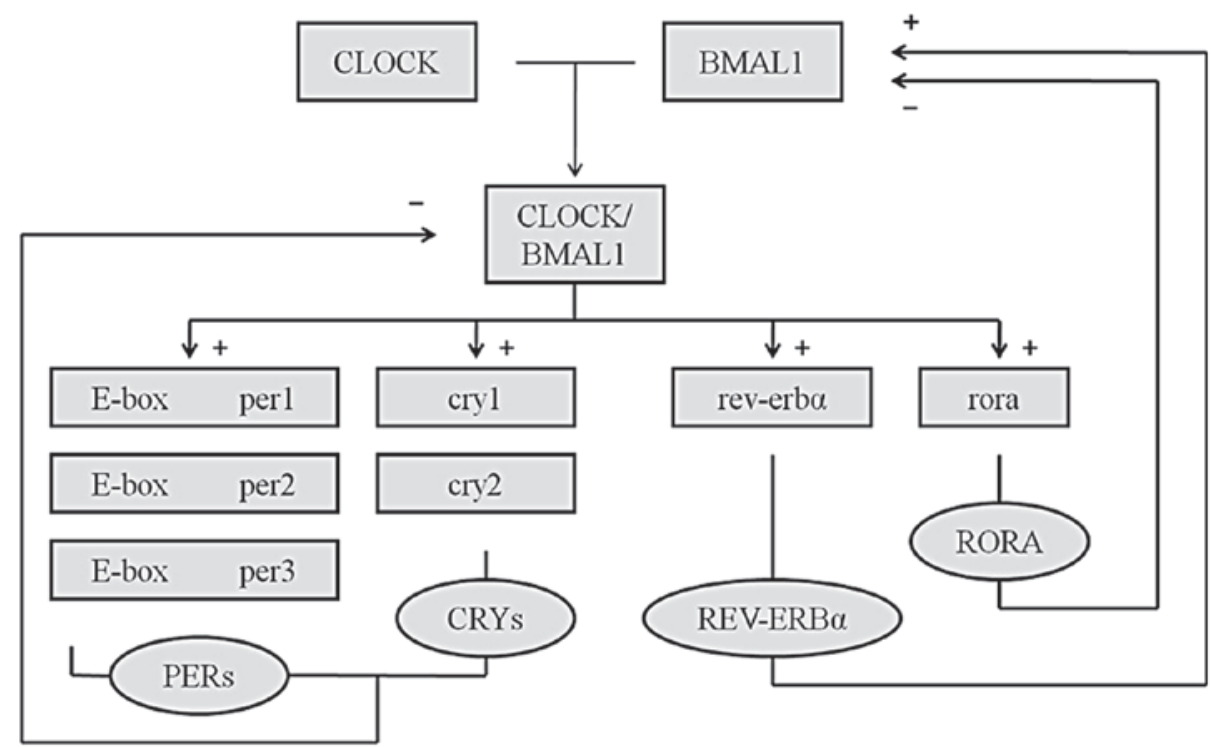

Figure 1. The Circadian Locomotor Output Cycles Kaput (CLOCK) gene feedback pathway. The CLOCK/BMAL1 complex activates transcription of per-, cry-, rora and rev-erb $\alpha$ genes. PER and CRY proteins inhibit (-) the transcription of the CLOCK/BMAL1 complex. REV-ERB $\alpha$ inhibits and RORA promotes (+) bmall transcription. Figure reproduced from references $(8,12)$. BMAL1, brain and muscle ARNT-like protein 1; PER, period circadian clock; CRY, cryptochrome; REV-ERB $\alpha$, nuclear receptor subfamily 1, group D, member 1; and RORa, retinoic acid-related orphan nuclear receptor a.

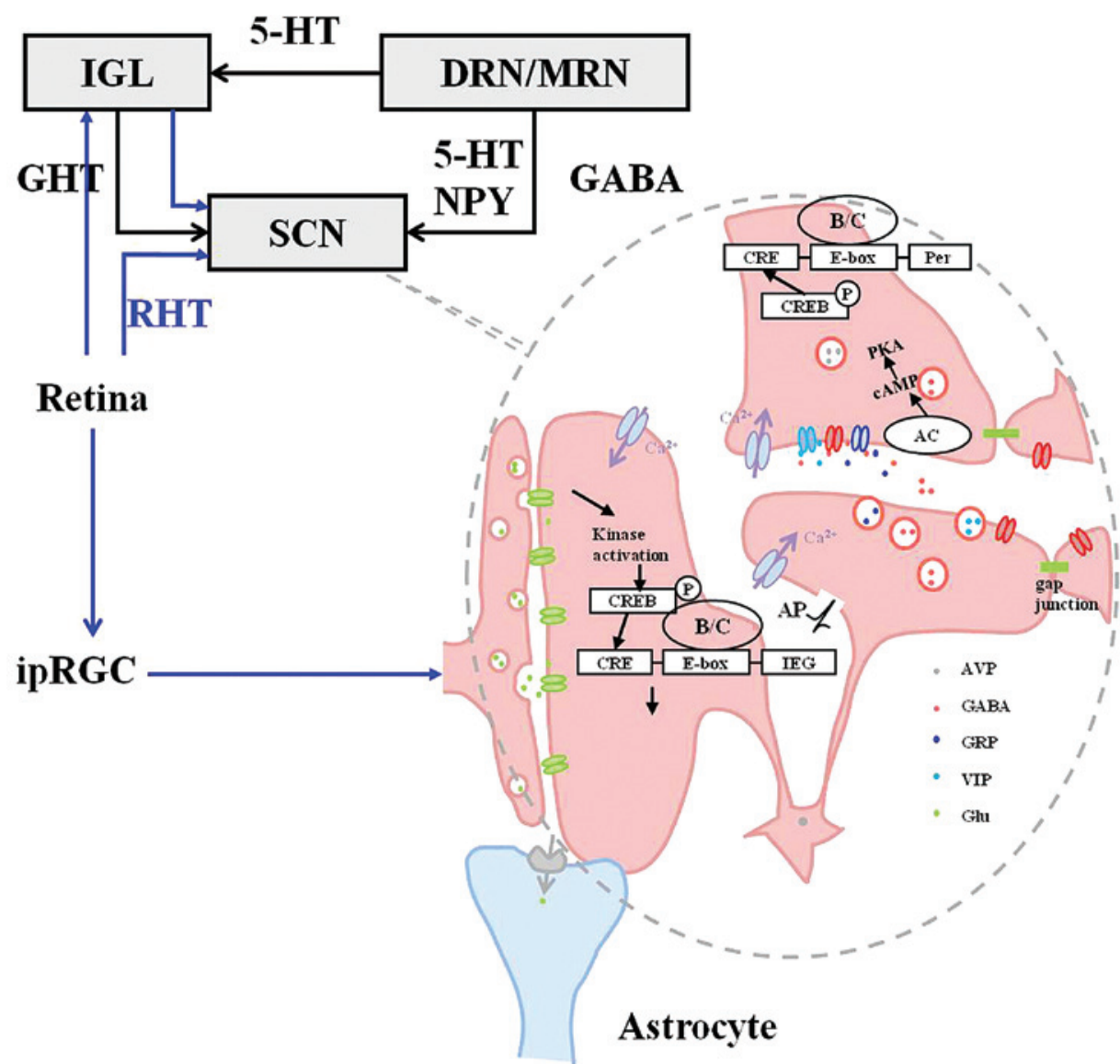

Figure 2. Intracellular mechanism of Circadian Locomotor Output Cycles Kaput (CLOCK) gene regulation in the suprachiasmatic nucleus (SCN). Blue arrows, optical signal afferent pathway to the SCN (i); and black arrows, non-optical signal afferent pathway (ii). i) Photic input to the SCN. Intrinsically photosensitive retinal ganglion cells (ipRGCs) project into the SCN via the retinohypothalamic tract (RHT). Presynaptic membranes release glutamate (Glu), leading to kinase activation and a series of $C L O C K$ gene expression alterations, promoting expression of immediate early genes (IEG). Glu is introduced from the synaptic cleft into the cells by Glu transporters locating on the astrocyte membrane; and ii) photic input. IGL, intergeniculate leaflet; DRN, dorsal raphe nucleus; MRN, median raphe nucleus; GHT, geniculohypothalamic tract; 5-HT, serotonin; NPY, neuropeptide Y; GABA, $\gamma$-aminobutyric acid; cAMP, cyclic adenosine monophosphate; CRE, cAMP-response element; CREB, CRE-binding protein; B/C, brain and muscle ARNT-like protein 1 (BMAL1)/CLOCK; AP, action potential; AVP, arginine vasopressin; GRP, gastrin-releasing peptide; VIP, vasointestinal peptide; AC, adenylate cyclase; and PKA, protein kinase A. Figure reproduced from reference (19). 

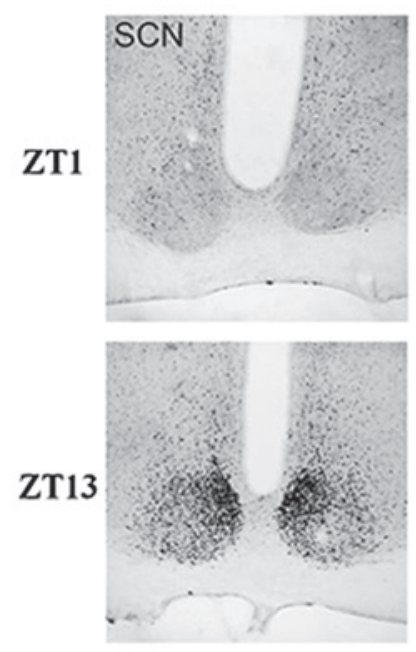
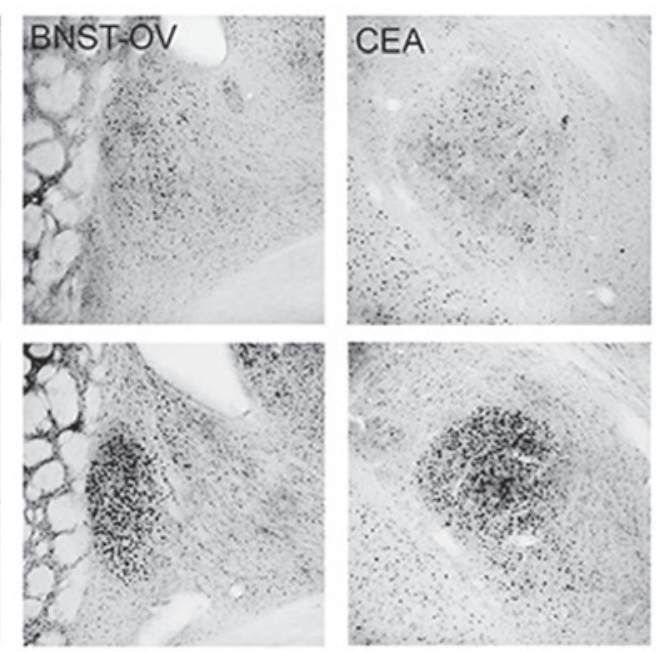
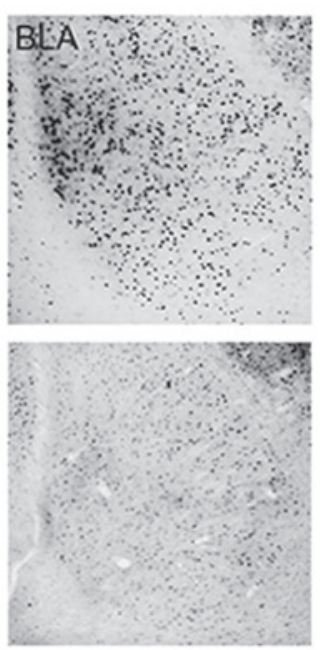
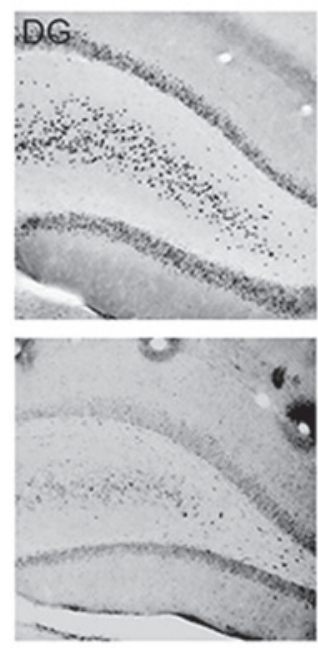

Figure 3. Period circadian clock 2 protein expression, detected by immunocytochemical staining in the suprachiasmatic nucleus (SCN) and the limbic system at zeitgeber times (ZT) 1 and 13, where ZT1, day time, start of lighting; and ZT13, night time, end of lighting. BNST-OV, oval nucleus of the bed nucleus of the stria terminalis; CEA, central nucleus of the amygdala; BLA, basolateral amygdala (400x400 $\mu \mathrm{m}$ frame); and DG, dentate gyrus (200x400 $\mu \mathrm{m}$ frame). Figure reproduced from reference (26).

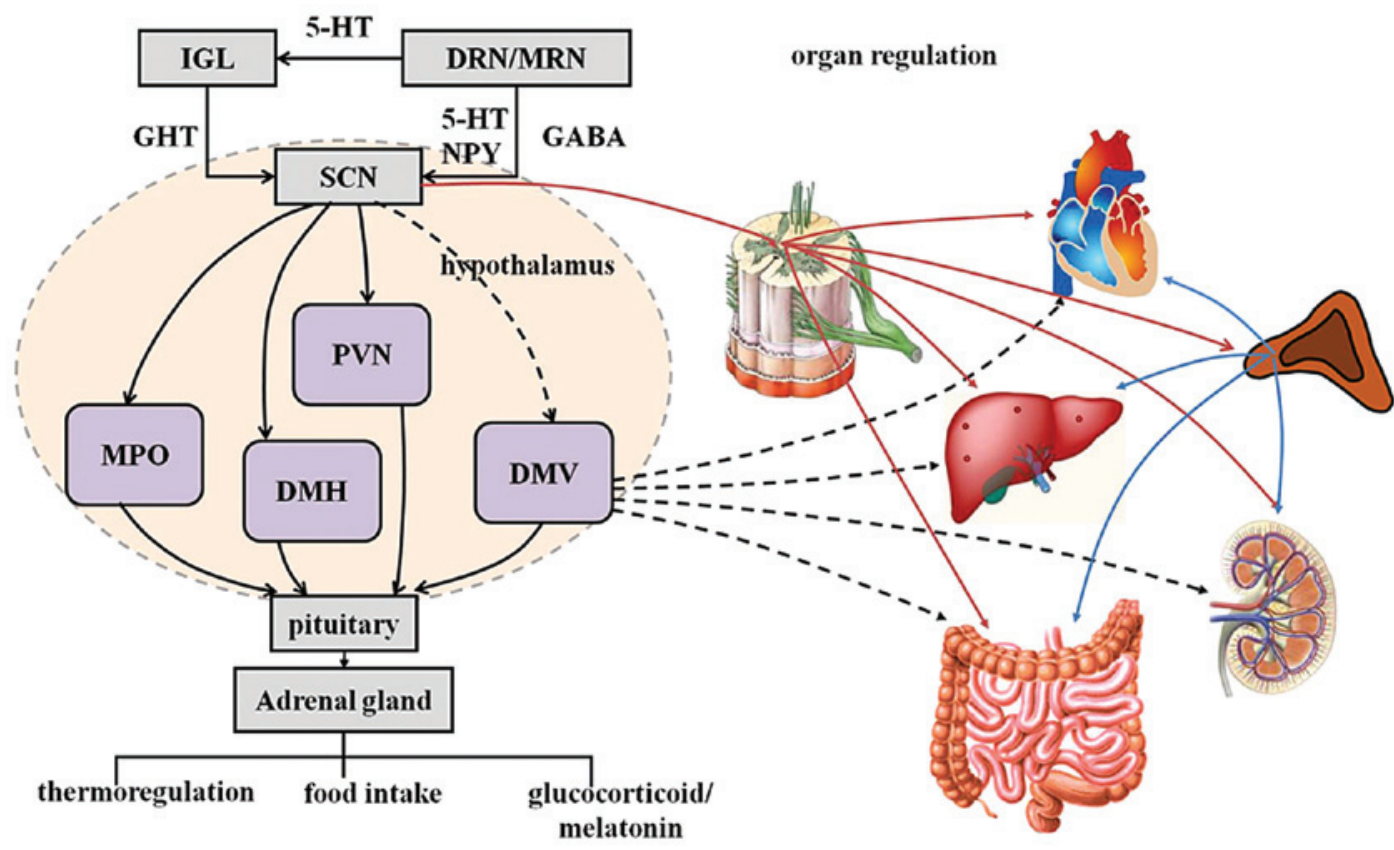

Figure 4. Role of the hypothalamus-pituitary-adrenal (HPA) axis on visceral organ regulation modulated by the suprachiasmatic nucleus (SCN). The Circadian Locomotor Output Cycles Kaput (CLOCK) gene and the HPA axis affect each other. The CLOCK gene is affected by light stimulation and regulates HPA axis excitability in the central nervous system, leading to rhythmic glucocorticoid release. In the peripheral nervous system, $C L O C K$ is mainly expressed in the adrenal gland and other HPA axis-related regions. The pituitary is regulated by the CLOCK gene in the central nervous system (via the sympathetic nervous system) and rhythmically regulated by glucocorticoids secreted by the adrenal gland. Glucocorticoids exert a feedback effect, likely involving other genes related to the biological functions of CLOCK. Black dashed lines, parasympathetic nerves; red solid lines, sympathetic nerves; blue solid lines, glucocorticoids. IGL, intergeniculate leaflet; DRN, dorsal raphe nucleus; MRN, median raphe nucleus; GHT, geniculohypothalamic tract; 5-HT, serotonin; NPY, neuropeptide Y; GABA, $\gamma$-aminobutyric acid; PVN, paraventricular nucleus; MPO, medial preoptic region; DMH, dorsomedial nucleus of hypothalamus; and DMV, dorsal motor nucleus of the vagus nerve.

exogenous glucocorticoids profoundly affects rhythmic genes in these regions. Moreover, glucocorticoid effects on the limbic system are regulated by CRF, a promoter of the HPA axis, and HPA axis activation affects numerous surrounding target organs $(29,38,39)$. Following bilateral adrenal gland removal, rhythmic PER2 expression in the BNST and CeA disappears, while PER2 expression in the basolateral amygdala and DG is unaffected $(16,17)$. Administering corticosterone in drinking water restores rhythmic PER2 expression in the BNST and $\mathrm{CeA}$, while persistent subcutaneous pumping is ineffective (40), suggesting that glucocorticoid signaling is likely an important factor for regulating rhythmic PER2 expression in these brain regions.

In summary, the per 2 gene presents region-specific rhythmicity in the central nervous system. Target organs exhibit circadian rhythms through optical signal integration, 


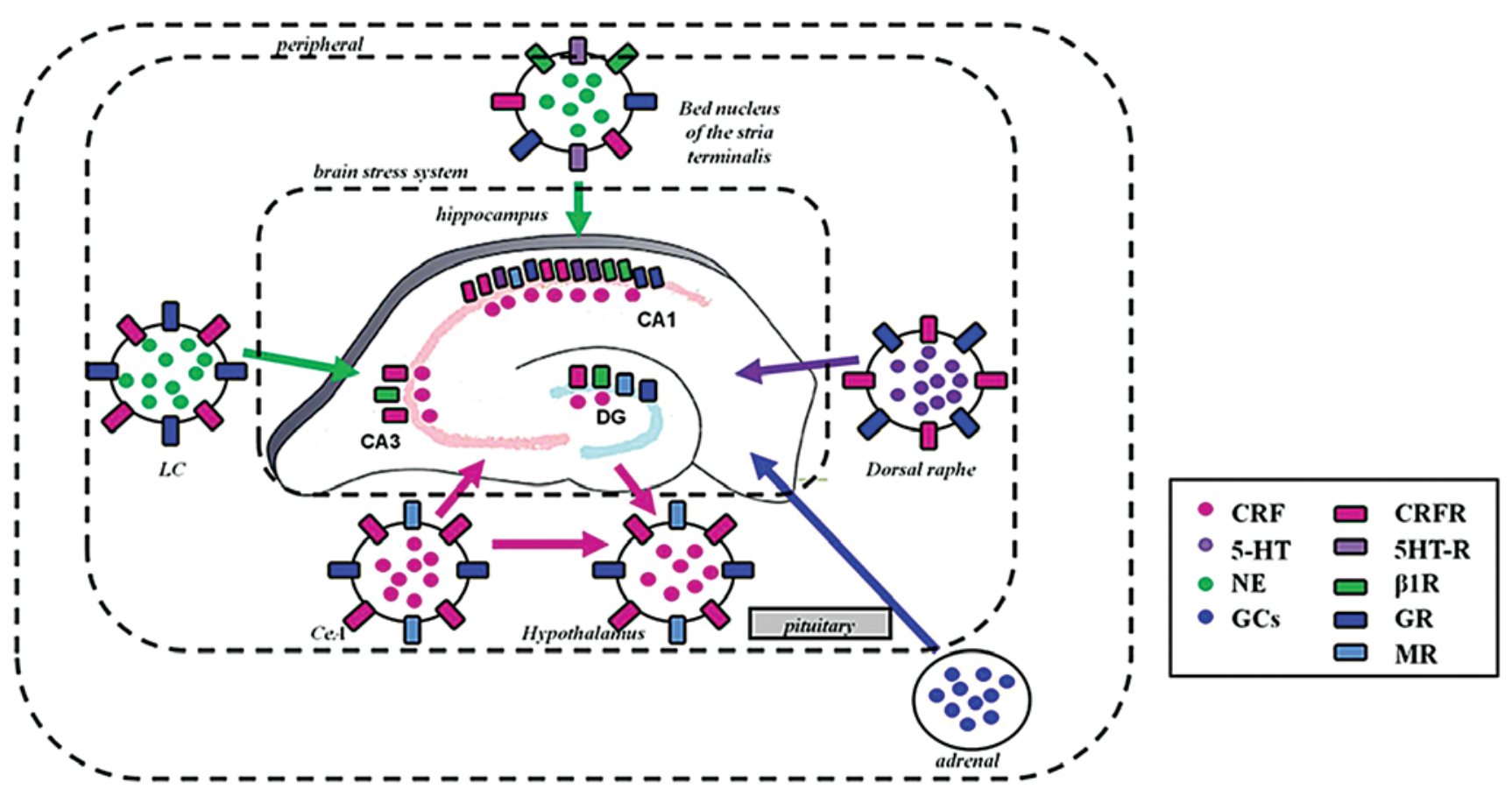

Figure 5. Role of glucocorticoids (GC) and other neurotransmitters on corticotropin-releasing factor (CRF) and the hypothalamus-pituitary-adrenal (HPA) axis. Upon external stress stimulation, the amygdala is activated first, followed by CRF neurons overexpressed in the amygdala and leading to increased CRF release in the hypothalamic paraventricular nucleus. Simultaneously, norepinephrine (NE) overexpression is detected in the locus coeruleus (LC) and the bed nucleus of the stria terminalis. Serotonin (5-HT) release increases in the dorsal raphe nucleus, resulting in elevated excitability of the HPA axis, increased GC release in surrounding tissues, and a visceral rhythmic motion disorder. The combination of GC and GC receptors in the hippocampus inhibits CRF overexpression in the amygdala and the hypothalamus, resulting in inhibition of the HPA axis hyperactivity. CeA, central nucleus of the amygdala; DG, dentate gyrus; CA, cornu Ammon; CRFR, cotricotropin-releasing factor receptor; 5HT-R, hydroxytryptamine receptor; $\beta 1 \mathrm{R}, \beta 1$-adrenergic receptors; GR, glucocorticoid receptor; MR, mineralocorticoid receptor.

CRF and related transmitters in the stress-response system, and regulation of the HPA axis excitability. Glucocorticoids and glucocorticoid receptors play a role in the negative feedback-type regulation of the period circadian clock 2 mRNA and protein and on the HPA axis in the limbic system, but the mechanism that the limbic system participates in requires further investigation.

\section{The PER2-hypothalamus-pituitary-adrenal (HPA) axis interaction regulates visceral activities}

Per2 gene regulation on the HPA axis. Optical signals activate the CLOCK gene in the SCN. The CRF/arginine-vasopressin immunoreactive neuronal projection to the hypothalamic paraventricular nucleus causes hypothalamic CRF release and leads to activation of the HPA axis, inducing rhy thmic release of glucocorticoids in the adrenal gland (41-43) (Fig. 5). CRF mRNA expression in the paraventricular nucleus synchronizes with per 2 expression in the SCN (44). Rhythmic per 2 expression patterns are not similar in the different parts of the HPA axis, and specifically in the hypothalamus (except the $\mathrm{SCN}$ ), and the pituitary and adrenal glands, with per 2 expression patterns being reversed between other regions of the hypothalamus and SCN. Similar per 2 expression patterns are found between the pituitary gland, adrenal gland and SCN. Following SCN injury, the function of the HPA axis is attenuated, and the release of adrenocorticotropic hormone from the pituitary gland is reduced, but rhythmicity is maintained. Moreover, the rhythmicity of glucocorticoid release from the adrenal gland is unaffected by the central nervous system. Therefore, rhythmic secretion of the adrenocorticotropic hormone from the pituitary gland is possibly associated with a negative feedback effect of glucocorticoids in the peripheral nervous system (45).

Rhythmic glucocorticoid release, mediated by the $C L O C K$ gene within the central nervous system, plays an important role in the regulation of circadian adaptability. The SCN mediates autonomic nerve excitability, altering the sensitivity of the adrenal cortex to the adrenocorticotropic hormone and rhythmically released glucocorticoids from the adrenal gland $(41-43,46)$. Previous experiments detected no significant difference in baseline levels of serum glucocorticoids between per2 gene knock-out and wild-type mice. However, the circadian rhythms were absent in per2 knock-out mice, while the pituitary gland-released adrenocorticotropic hormone lost its original circadian rhythm $(47,48)$. Another study demonstrated that in $p e r 2^{\text {Brdm } 1 / \text { rryl-/ }}$ mice, glucocorticoid biosynthesis is disrupted in both adrenal glands (46), suggesting the per2 gene controls glucocorticoid synthesis in adrenal glands, which may also be regulated by adrenocorticotropic hormone that is rhythmically released from the pituitary gland. Thus, it is assumed that in the central nervous system, the per 2 gene can be regulated by both optical signals and the rhythmic secretion of glucocorticoids (products of the HPA axis), but its regulation may be either dependent or independent of the HPA axis. Regulation of the per 2 gene within the adrenal gland by secretory products from other parts of the HPA axis (for example, adrenocorticotropic hormone released from the 
pituitary gland), also affects the circadian rhythm of organs in the peripheral nervous system (Fig. 5).

Effects of the HPA axis on the per2 gene. The HPA axis strongly affects per 2 expression within the majority of organs by regulating glucocorticoid release, but minimally affects per2 expression in the central nervous system. This is due to reduced glucocorticoid levels in the central nervous system and decreased co-expression of PER2 and glucocorticoid receptors in the BNST and CeA (49). Therefore, the circadian rhythm of the $C L O C K$ gene in the central nervous system is independent of changes in the surrounding environment. However in the peripheral nervous system, CLOCK gene expression exhibits rhythmic phase shifts during adjustment to surrounding changes, and is restored to normal rhythmic levels through regulation by glucocorticoid negative feedback in the central nervous system. Within organs, glucocorticoid-mediated intermittent phasic resetting are associated with alterations in the $C L O C K$ gene rhythmicity, induced by adjustments to stress within each organ (49-51).

\section{Clinical significance of the PER2-HPA axis interaction on regulation of visceral rhythmic movement}

Visceral rhythmic movements stimulated by the environmental stressors and show rhythmic motion alterations. The HPA axis integrates limbic system information and regulates target organs (Figs. 4 and 5). The PER2 effects on visceral activities include tissue metabolism and organ movement. The PER2-HPA axis interaction is important for studies of metabolic syndrome, functional intestinal tract disease, hepatic metabolism and immune system-related diseases.

Metabolic syndrome. Previous studies examining PER2 effects on calorie intake and metabolism have shown that the food intake rate is delayed and the weight is increased in per 2 gene knock-out mice. The weight of per 2 knock-out mice provided with the same food intake daily as wild-type mice was similar to that of high-fat diet-fed mice suffering from an orexin secretion disorder $(52,53)$. This disorder is associated with PER2 effects on glucocorticoid synthesis, release and metabolism. CRF participates in the negative feedback effect of glucocorticoids on PER2. A number of observations support that PER2 is involved in metabolic disease: i) hypercortisolism (or Cushing's syndrome) causes central obesity; ii) bilateral adrenal gland removal induces a reduction in food intake and weight due to the permanent absence of cortisol, which regulates per 2 in the organs and provides negative feedback to the hypothalamus, hippocampus and other brain sites in the CNS; iii) cortisol plays an important role in maintaining metabolic balance, including regulation of food intake and insulin levels (54); iv) following adrenalectomy, cortisol function is disrupted, while upon hypophysectomy, the function of the HPA axis is disrupted. These disorders can lead to cortisol release, circadian disappearance, irregular food intake and even body weight gain (55); and v) Cushing's syndrome patients experience anxiety, one of the manifestations of acute or chronic stress, which can result in increased cortisol release, rhythmic destruction and reduced CRF levels in the hypothalamus and the pituitary gland. Thus, patients are eager to have high-calorie and -fat food, with the associated reduction in hypothalamic CRF release, reducing stress-induced anxiety-like behavior (56). Short-term damage in the rhythmicity of cortisol release induces increases in postprandial blood sugar, serum insulin levels and mean arterial blood pressure, with significant reductions in the level of leptin and sleep efficiency, and even complete loss of control of the rhythmic movement of the organs by cortisol (57).

Altogether, restoring the cortisol-PER2 interaction may provide a novel research direction and method for the treatment of Cushing's syndrome in the clinic. However, a number of issues need to be addressed in this direction. For example, it is difficult to observe the rhythmic cortisol pattern in living animals or humans. Thus, whether damage in the cortisol circadian rhythms results in obesity, or obesity causes cortisol circadian rhythms disturbance remains to be clarified.

Irritable bowel syndrome (IBS). Time differences cause resets in per 2 gene expression in the intestinal tract, leading to intestinal dysfunctions including abdominal pain, constipation and diarrhea, which are the main causes and symptoms of IBS (58-62). With regards to intestinal functional regulation in the peripheral nervous system, the per 2 gene is mainly expressed in the myenteric plexus of the intestinal tract, where neurotransmitters promoting intestinal movement are synthesized (8). The HPA axis plays an important regulatory role in intestinal movement. Rhythmic CRF release in the hypothalamic paraventricular nucleus regulates intestinal rhythmic motion. The stress reaction, via CRF neuronal activation in the HPA axis and the limbic system, regulates autonomic nervous system excitability and affects intestinal movement (Figs. 4 and 5). Thus, adrenocorticotropic hormone levels are increased in the HPA axis, rhythmicity decreased and the colon cortisol level increased (63). It is important to study the PER2-HPA axis interaction and its roles in IBS for the following reasons: i) in recent years, the rapidly developing Asian economy has been strongly associated with an increased incidence of IBS, with fast-paced work, sleep, food and drink appearing as main stress factors (64); ii) patients with IBS show sleep disturbances $(65,66)$, which are a hallmark symptom of CLOCK gene abnormalities in the central nervous system $(60,67,68)$; iii) a clinical randomized and placebo-controlled trial demonstrated that melatonin, an important regulatory peptide of the circadian rhythm, can reduce intestinal symptoms in IBS patients (69); and v) there are correlations between intestinal sensitivity increased induced abdominal pain and circadian disorder in IBS patients (70). In addition, electroacupuncture noticeably improves sleep quality in patients with sleep disturbances (71), providing therapeutic evidence for a sleep-related functional disorder in other organs.

\section{Perspectives}

The PER2 protein and the HPA axis interact via glucocorticoids to regulate visceral circadian activities. Glucocorticoids in adjacent organs are regulated by rhythmic CRF alterations in the HPA axis and its response to stress. In the HPA axis, CRF is regulated by the limbic system, 
including the BNST, CeA and hippocampus, and shows a direct or indirect connection to rhythmic alterations in PER2 expression affecting the morphology and behavior of emotion and food intake. In general, the HPA axis is a mediator of the effects of PER2 on target organs. The limbic system integrates stress-related (CRF, stress-related neurotransmitters and neuropeptides) and optical signals, regulating the HPA axis excitability. To date, numerous clinical and fundamental research studies have examined the PER2-HPA axis interaction in the context of metabolic syndrome and IBS. Although the exact mechanism underlying the contributions of the PER2-HPA axis interaction in these disorders remains unclear, associations with the limbic system deserve further investigation. Future studies of the per 2 gene need to focus on the following aspects: i) CRF rhythmic expression in the hypothalamus and pituitary, rhythmic expression of ACTH in the pituitary and cortisol rhythmic changes in the adrenal gland, are included in the HPA axis circadian rhythm. Previous studies have focused on the effects of SCN on the PER2-HPA axis interaction, including local expression and biological function of PER2 in the SCN, the effect of PER2 in the regulation of the circadian rhythm in target organs, and the glucocorticoids negative feedback to the brain areas in the limbic systems (41-44). A few studies have identified an interaction between the limbic system and per 2 expression in the $\operatorname{SCN}(23,24)$. Conversely, the limbic system, in particular the hippocampus and amygdala, plays an important role in HPA axis priming and regulation. Therefore, the limbic system may be an important connection between the HPA axis and the cerebral cortex in the mediation of the PER2 effects (27); ii) intracellular signaling mechanisms underlying the hypothalamic CRF-PER2 interaction. CRF activates the HPA axis and is regulated by the hippocampus and amygdala (25). Pharmacological studies have confirmed that there is a correlation between the biological function of PER2 and CRF, and although morphological studies have not identified a direct correlation, it is likely to be mediated by intracellular signaling, although the exact molecular mechanism is unknown (31-33, 10, 35); and iii) studies examining the contribution of the per 2 gene in various metabolic, neuroendocrine and neuroimmunological diseases (22-24). The per2 regulatory effect on HPA axis circadian rhythms and its roles on the autonomic nervous system, emotion and behavior, in functional disorders of autonomic nerve-induced diseases (including functional gastrointestinal disorders and heart disease), various stresses (including psychological, social and post-trauma acute and chronic stress), cognitive function and behavioral alterations. A breakthrough of the central mechanism of per2 gene interacting with the HPA axis is in urgent need to deepen the understanding of per2 circadian rhythm physiopathologically and contribute to the treatment of per 2 rhythm disturbance related diseases.

\section{Acknowledgements}

This study was supported by the grant no. 81202735 (2012) from the National Natural Science Foundation of China, and the Excellent Backbone Teacher Program of Qinglan Engineering of the Jiangsu Province in China.

\section{References}

1. Li MD, Ruan HB, Hughes ME, et al: O-GlcNAc signaling entrains the circadian clock by inhibiting BMAL1/CLOCK ubiquitination. Cell Metab 17: 303-310, 2013.

2. Korencic A, Bordyugov G, Kosir R, Rozman D, Golicnik M and Herzel H: The interplay of cis-regulatory elements rules circadian rhythms in mouse liver. PLoS One 7: e46835, 2012.

3. Prendergast BJ, Cisse YM, Cable EJ and Zucker I: Dissociation of ultradian and circadian phenotypes in female and male Siberian hamsters. J Biol Rhythms 27: 287-298, 2012.

4. Granados-Fuentes D, Ben-Josef G, Perry G, Wilson DA, Sullivan-Wilson A and Herzog ED: Daily rhythms in olfactory discrimination depend on clock genes but not the suprachiasmatic nucleus. J Biol Rhythms 26: 552-560, 2011.

5. Valnegri P, Khelfaoui M, Dorseuil O, et al: A circadian clock in hippocampus is regulated by interaction between oligophrenin-1 and Rev-erba. Nat Neurosci 14: 1293-1301, 2011.

6. Amir S and Stewart J: Motivational modulation of rhythms of the expression of the clock protein PER2 in the limbic forebrain. Biol Psychiatry 65: 829-834, 2009.

7. Amir S and Stewart J: Behavioral and hormonal regulation of expression of the clock protein, PER2, in the central extended amygdala. Prog Neuropsychopharmacol Biol Psychiatry 33: 1321-1328, 2009.

8. Yamaguchi M, Kotani K, Sakane N, et al: The CLOCK 3111T/C SNP is associated with morning gastric motility in healthy young women. Physiol Behav 107: 87-91, 2012.

9. Kucera N, Schmalen L, Hennig S, et al: Unwinding the differences of the mammalian PERIOD clock proteins from crystal structure to cellular function. Proc Natl acad Sci USA 109: 3311-3316, 2012.

10. Hood S, Cassidy P, Cossette MP, et al: Endogenous dopamine regulates the rhythm of expression of the clock protein PER2 in the rat dorsal striatum via daily activation of D2 dopamine receptors. J Neurosci 30: 14046-14058, 2010.

11. Wang Y, Yao Q and Chen KP: Progress of studies on family members and functions of animal bHLH transcription factors. Yi Chuan 32: 307-330, 2010 (In Chinese).

12. Swanson G, Forsyth CB, Tang Y, et al: Role of intestinal circadian genes in alcohol-induced gut leakiness. Alcohol Clin Exp Res 35: 1305-1314, 2011.

13. Konturek PC, Brzozowski T and Konturek SJ. Gut clock: implication of circadian rhythms in the gastrointestinal tract. J Physiol Pharmacol 62: 139-150, 2011.

14. Manfredini R and Portaluppi F: Night shift and impaired endothelial function: circadian out-of-synch may play a role. Int J Cardiol 154: 94-95, 2012.

15. Tonsfeldt KJ and Chappell PE: Clocks on top: the role of the circadian clock in the hypothalamic and pituitary regulation of endocrine physiology. Mol Cell Endocrinol 349: 3-12, 2012.

16. Harbour VL, Robinson B and Amir S: Variations in daily expression of the circadian clock protein, PER2, in the rat limbic forebrain during stable entrainment to a long light cycle. J Mol Neurosci 45: 154-161, 2011.

17. Mueller AD, Mear RJ and Mistlberger RE: Inhibition of hippocampal neurogenesis by sleep deprivation is independent of circadian disruption and melatonin suppression. Neuroscience 193: 170-181, 2011.

18. Pezuk P, Mohawk JA, Wang LA and Menaker M: Glucocorticoids as entraining signals for peripheral circadian oscillators. Endocrinology 153: 4775-4783, 2012.

19. Albrecht U: Timing to perfection: the biology of central and peripheral circadian clocks. Neuron 74: 246-260, 2012.

20. Glass JD, Brager AJ, Stowie AC and Prosser RA: Cocaine modulates pathways for photic and nonphotic entrainment of the mammalian SCN circadian clock. Am J Physiol Regul Integr Comp Physiol 302: R740-R750, 2012.

21. Ripperger JA and Albrecht U: The circadian clock component PERIOD2: from molecular to cerebral functions. Prog Brain Res 199: 233-245, 2012.

22. Hagenauer MH and Lee TM: The neuroendocrine control of the circadian system: Adolescent chronotype. Front Neuroendocrinol 33: 211-229, 2012.

23. Jiang WG, Li SX, Zhou SJ, Sun Y, Shi J and Lu L: Chronic unpredictable stress induces a reversible change of PER2 rhythm in the suprachiasmatic nucleus. Brain Res 1399: 25-32, 2011.

24. Ogawa Y, Koike N, Kurosawa G, Soga T, Tomita M and Tei H: Positive autoregulation delays the expression phase of mammalian clock gene Per2. PLoS One 6: e18663, 2011. 
25. Myung J, Hong S, Hatanaka F, Nakajima Y, De Schutter E and Takumi T. Period coding of Bmall oscillators in the suprachiasmatic nucleus. J Neurosci. 32: 8900-8918, 2012.

26. Duong HA, Robles MS, Knutti D and Weitz CJ: A molecular mechanism for circadian clock negative feedback. Science 332: 1436-1439, 2011

27. Perrin JS, Segall LA, Harbour VL, Woodside B and Amir S: The expression of the clock protein PER2 in the limbic forebrain is modulated by the estrous cycle. Proc Natl Acad Sci USA 103: $5591-5596,2006$.

28. Maras PM and Baram TZ: Sculpting the hippocampus from within: stress, spines, and CRH. Trends Neurosci 35: 315-324, 2012.

29. Carrasco J, Marquez C, Nadal R, Tobena A, Fernandez-Teruel A and Armario A: Characterization of central and peripheral components of the hypothalamus-pituitary-adrenal axis in the inbred Roman rat strains. Psychoneuroendocrinology 33: 437-445, 2008.

30. Hill MN, Patel S, Campolongo P, Tasker JG, Wotjak CT and Bains JS: Functional interactions between stress and the endocannabinoid system: from synaptic signaling to behavioral output. J Neurosci 30: 14980-14986, 2010.

31. Costine BA, Oberlander JG, Davis MC, et al: Chronic anabolic androgenic steroid exposure alters corticotropin releasing factor expression and anxiety-like behaviors in the female mouse. Psychoneuroendocrinology 35: 1473-1485, 2010.

32. Silberman Y, Matthews RT and Winder DG: A corticotropin releasing factor pathway for ethanol regulation of the ventral tegmental area in the bed nucleus of the stria terminalis. J Neurosci 33: 950-960, 2013.

33. Cruz MT, Herman MA, Kallupi $M$ and Roberto $M$ : Nociceptin/orphanin FQ blockade of corticotropin-releasing factor-induced gamma-aminobutyric acid release in centra amygdala is enhanced after chronic ethanol exposure. Biol Psychiatry 71: 666-676, 2012.

34. Malloy JN, Paulose JK, Li Y and Cassone VM: Circadian rhythms of gastrointestinal function are regulated by both central and peripheral oscillators. Am J Physiol Gastrointest Liver Physiol 303: G461-G473, 2012.

35. Gaszner B, Van Wijk DC, Korosi A, Jozsa R, Roubos EW and Kozicz T: Diurnal expression of period 2 and urocortin 1 in neurones of the non-preganglionic Edinger-Westphal nucleus in the rat. Stress 12: 115-124, 2009.

36. Huang TS, Ruoff P and Fjelldal PG: Effect of continuous light on daily levels of plasma melatonin and cortisol and expression of clock genes in pineal gland, brain, and liver in atlantic salmon postsmolts. Chronobiol Int 27: 1715-1734, 2010.

37. Kino T: Circadian rhythms of glucocorticoid hormone actions in target tissues: potential clinical implications. Sci Signal 5: pt4, 2012. doi: 10.1126/scisignal.2003333.

38. Schulkin J: Evolutionary conservation of glucocorticoids and corticotropin releasing hormone: behavioral and physiological adaptations. Brain Res 1392: 27-46, 2011.

39. Son GH, Chung S and Kim K: The adrenal peripheral clock: glucocorticoid and the circadian timing system. Front Neuroendocrino 32: 451-465, 2011

40. Nader N, Chrousos GP and Kino T: Interactions of the circadian CLOCK system and the HPA axis. Trends Endocrinol Metab 21: 277-286, 2010.

41. Charmandari E, Chrousos GP, Lambrou GI, et al: Peripheral CLOCK regulates target-tissue glucocorticoid receptor transcriptional activity in a circadian fashion in man. PLoS One 6: e25612 2011.

42. Lilley TR, Wotus C, Taylor D, Lee JM and de la Iglesia HO Circadian regulation of cortisol release in behaviorally split golden hamsters. Endocrinology 153: 732-738, 2012.

43. So AY, Bernal TU, Pillsbury ML, Yamamoto KR and Feldman BJ Glucocorticoid regulation of the circadian clock modulates glucose homeostasis. Proc Natl Acad Sci USA 106: 17582-17587, 2009.

44. Girotti M, Weinberg MS and Spencer RL: Diurnal expression of functional and clock-related genes throughout the rat HPA axis: system-wide shifts in response to a restricted feeding schedule Am J Physiol Endocrinol Metab 296: E888-E897, 2009.

45. Helms CM, McClintick MN and Grant KA: Social rank, chronic ethanol self-administration, and diurnal pituitary-adrenal activity in cynomolgus monkeys. Psychopharmacology (Berl) 224: 133-143, 2012.

46. Chung S, Son GH and Kim K: Adrenal peripheral oscillator in generating the circadian glucocorticoid rhythm. Ann NY Acad Sci 1220: 71-81, 2011.
47. Qu X, Metz RP, Porter WW, Neuendorff N, Earnest BJ and Earnest DJ: The clock genes period 1 and period 2 mediate diurnal rhythms in dioxin-induced Cypl A1 expression in the mouse mammary gland and liver. Toxicol Lett 196: 28-32, 2010.

48. Yang S, Liu A, Weidenhammer A, et al: The role of mPer2 clock gene in glucocorticoid and feeding rhythms. Endocrinology 150: 2153-2160, 2009.

49. Imanishi M, Yamamoto K, Yamada H, Hirose Y, Okamura H and Futaki S: Construction of a rhythm transfer system that mimics the cellular clock. ACS Chem Biol 7: 1817-1821, 2012.

50. Nebzydoski SJ, Pozzo S, Nemec L, Rankin MK and Gressley TF: The effect of dexamethasone on clock gene mRNA levels in bovine neutrophils and lymphocytes. Vet Immunol Immunopathol 138 183-192, 2010

51. Verwey M and Amir S: Variable restricted feeding disrupts the daily oscillations of Period 2 expression in the limbic forebrain and dorsal striatum in rats. J Mol Neurosci 46: 258-264, 2012.

52. Sherman H, Genzer Y, Cohen R, Chapnik N, Madar Z and Froy O: Timed high-fat diet resets circadian metabolism and prevents obesity. FASEB J 26: 3493-3502, 2012.

53. Milagro FI, Gomez-Abellan P, Campion J, Martinez JA, Ordovas JM and Garaulet M: CLOCK, PER2 and BMAL1 DNA methylation: association with obesity and metabolic syndrome characteristics and monounsaturated fat intake. Chronobiol Int 29: 1180-1194, 2012

54. Solomon MB, Sakai RR, Woods SC and Foster MT: Differential effects of glucocorticoids on energy homeostasis in Syrian hamsters. Am J Physiol Endocrinol Metab 301: E307-E316, 2011

55. Nyberg CH, Leonard WR, Tanner S, et al: Diurnal cortisol rhythms and child growth: exploring the life history consequences of HPA activation among the Tsimane'. Am J Hum Biol 24: 730-738, 2012.

56. Parylak SL, Cottone P, Sabino V, Rice KC and Zorrilla EP: Effects of CB1 and CRF1 receptor antagonists on binge-like eating in rats with limited access to a sweet fat diet: lack of withdrawal-like responses. Physiol Behav 107: 231-242, 2012.

57. Scheer FA, Hilton MF, Mantzoros CS and Shea SA: Adverse metabolic and cardiovascular consequences of circadian misalignment. Proc Natl Acad Sci USA 106: 4453-4458, 2009.

58. Hoogerwerf WA: Role of clock genes in gastrointestinal motility. Am J Physiol Gastrointest Liver Physiol 299: G549-G555, 2010.

59. Iwashina I, Mochizuki K, Inamochi Y and Goda T: Clock genes regulate the feeding schedule-dependent diurnal rhythm changes in hexose transporter gene expressions through the binding of BMAL1 to the promoter/enhancer and transcribed regions. J Nutr Biochem 22: 334-343, 2011

60. Wells MM, Roth L and Chande N: Sleep disruption secondary to overnight call shifts is associated with irritable bowel syndrome in residents: a cross-sectional study. Am J Gastroenterol 107: 1151-1156, 2012.

61. Vyas MV, Garg AX, Iansavichus AV, et al: Shift work and vascular events: systematic review and meta-analysis. BMJ 345: e4800, 2012

62. Nojkov B, Rubenstein JH, Chey WD and Hoogerwerf WA: The impact of rotating shift work on the prevalence of irritable bowel syndrome in nurses. Am J Gastroenterol 105: 842-847, 2010.

63. Heitkemper MM, Cain KC, Deechakawan W, et al: Anticipation of public speaking and sleep and the hypothalamic-pituitary-adrenal axis in women with irritable bowel syndrome. Neurogastroenterol Motil 24: 626-631, 2012.

64. Khan S and Chang L: Diagnosis and management of IBS. Nat Rev Gastroenterol Hepatol 7: 565-581, 2010.

65. Bellini M, Gemignani A, Gambaccini D, et al: Evaluation of latent links between irritable bowel syndrome and sleep quality. World J Gastroenterol 17: 5089-5096, 2011.

66. Hongo M: Epidemiology of FGID symptoms in Japanese general population with reference to life style. J Gastroenterol Hepatol 26 (Suppl 3): 19-22, 2011.

67. Burioka N, Koyanagi S, Endo M, et al: Clock gene dysfunction in patients with obstructive sleep apnoea syndrome. Eur Respir J 32: 105-112, 2008.

68. Miyamoto H, Nakamaru-Ogiso E, Hamada K and Hensch TK: Serotonergic integration of circadian clock and ultradian sleep-wake cycles. J Neurosci 32: 14794-14803, 2012.

69. Mozaffari S, Rahimi R and Abdollahi M: Implications of melatonin therapy in irritable bowel syndrome: a systematic review. Curr Pharm Des 16: 3646-3655, 2010.

70. Enck P, Kaiser C, Felber M, et al: Circadian variation of rectal sensitivity and gastrointestinal peptides in healthy volunteers. Neurogastroenterol Motil 21: 52-58, 2009.

71. Freire AO, Sugai GC, Togeiro SM, Mello LE and Tufik S: Immediate effect of acupuncture on the sleep pattern of patients with obstructive sleep apnoea. Acupunct Med 28: 115-119, 2010. 